\title{
INITIAL STEPS TO VIBRATIO V CONTROL OF A CONCRETE PUMP
}

\author{
Dirk Nissing, Werner Bernzen anıl Torsten Wey \\ University of Duisburg, Faculty of Mechanical Engineering, \\ Department of Measurement and Control (Pr,f. Dr.-Ing. H. Schwarz), \\ D-47048 Duisburg, Gerriany \\ Phone: ++49 (203) $379: 646$ \\ Fax: ++49 (203) 37931$) 27$ \\ Email: nissing@uni-duisburg.de
}

\begin{abstract}
Concrete pump robots are used for very wite operating ranges, so elastic deformations of the links must be taken into account. Cincrete pumps demand actuators with a high power density, able to produce large forces $v$ hich can be achieved by the use of hydraulically driven actuators. Hydrostatic differertial cylinders are widely used because of their simple construction. For multilink flexit le robots actuated by hydrostatic differential cylinders it is not possible to establish an ac zurate analytical model and this makes vibration damping control for the endeffector diffi : ult. A recent model independent approach to active damping control is used and prese ited in this paper. The control concept is described, where the actuators move as virtual spring-damper elements and this concept is used for vibration control of a concrete pump.
\end{abstract}

Keywords: concrete pump, flexible links, vibration damping, hydraulic actuators, nonlinear control.

\section{INTRODUCTION}

Conventional robots are designed with massive links, so that the elastic deformations can be neglected. In relationship to the weight of the robot, the payload is modest. To control the endeffector position of these systems is relatively easy, because only easily calculated parameters (inertia, mass and geometry) are needed [3]. The rigid construction leads to an overdimensioning of the loaded devices.

Two cases are obvious where elastic deformations have to be taken into account: heavy loads and very wide operating ranges. Concrete pump robots, depicted in figure 1 , for distributing concrete in the construction industry are examples for such wide operating ranges. The elastic deformations in the arms cannot be neglected. Rigid links will make the concrete pump too heavy, so that it is not possible to transport it from one place to another.

With partial differential equations it is possible to formulate a mathematical model for a single link flexible robot [4], but with an increasing number of links the differential equation system grows rapidly. Due to the high forces involved and the demand for light weight actuators in relation to the payload,

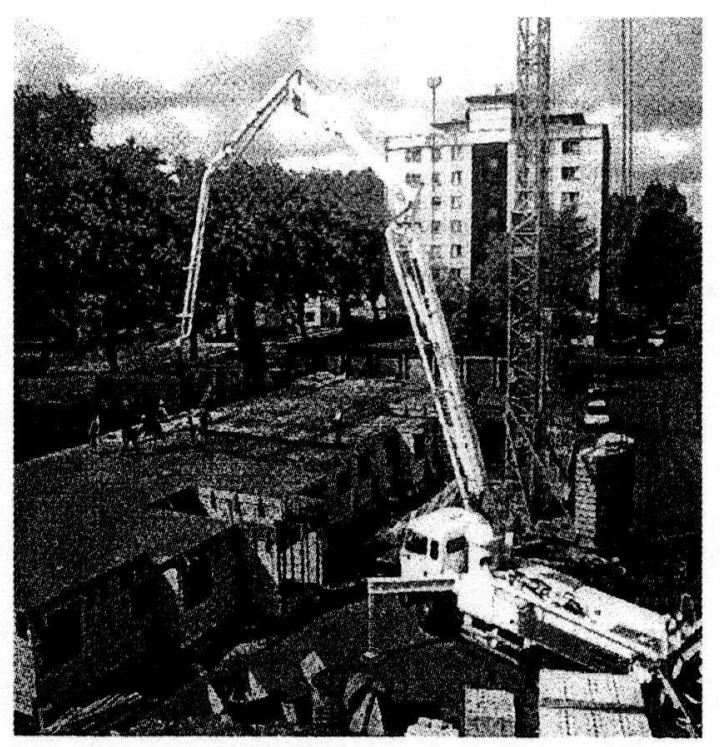

Figure 1. Concrete pumping robot

hydraulic actuators are the only viable drives [1]. Different al cylinders are the most commonly implemented actuators and they are controlled by proportional- or servovalves. Hydraulically driven 
cylinders have significant eigendynamics [5], which must be regarded in the controller design.

The development and the trial of a control concept to reduce the vibration of the endeffector builds the contents of this paper. The approach involves controlling the actuator of a concrete pump (a hydrostatic differential cylinder), so that it behaves like a virtual passive mechanical springdamper-element [2]. The advantage of the passive system is that energy is only dissipated, so that the device can never destabilize a system. Furthermore an analytical model of the robot, which practically never exist for complex systems, is not necessary. The new parameters of the virtual spring-damperelement (spring stiffness and damping constant) can be adjusteted experimentally. The more known about the system, the better these parameters may be selected to meet the optimum vibration damping of the boom. The new input of the controlled system is a virtual variation of the spring base.

The paper is organized as follows. The second section introduces the basic concept of vibration damping with a virtual spring-damper-element and how to apply this concept to a differential cylinder. Section 3 contains the velocity control concept of hydrostatic differential cylinders, which is necessary to realize the actuator as a spring-damper-element. The fourth section describes the structure and measurement constitution of the concrete pump, before the control concept is transfered to it. The fifth section shows results of the first vibration damping experiments. In the final section a conclusion and an outlook is given.

\section{BASIC CONCEPT OF VIBRATION DAMPING}

The basic concept for damping vibrations will be explained by an analytical model of a very simple flexible beam. The model of the flexible link with a tip mass (figure 2) can be established with the help of modal analysis. If Euler-Bemoulli beam theory can be used [7], the eigenfunctions

$$
\begin{gathered}
\phi_{0}(x)=C_{0} x \text { and } \\
\phi_{i}(x)=A_{i} \sin \left(\lambda_{i} x\right)+C_{i} \sinh \left(\lambda_{i} x\right), \quad 1 \leq i<\infty
\end{gathered}
$$

ensue. With the use of inner damping and a torque $T(t)$ in the joint, which is generated by the springdamper-element in the joint, a set of differential equations for an infinite number of modal coordinates $q_{i}(t)$ follows:

$$
\ddot{q}_{i}(t)+2 \delta_{i} \dot{q}(t)+\omega_{i}^{2} q_{i}(t)=\frac{\phi_{i}^{\prime}(0)}{J} T(t) .
$$

The output signal is calculated by

$$
y(x, t)=\sum_{i=0}^{\infty} \phi_{i}(x) q_{i}(t) .
$$

A statt space model resulting from eqs. (3) and (4) truncaled to three flexible modes is given by

$$
\dot{x}(t)=A x(t)+b T(t) \text { and } y(t)=c^{\mathrm{T}} x(t)
$$

with the state vector

$$
\boldsymbol{x}=\left[\begin{array}{llllllll}
l_{0} & q_{1} & q_{2} & q_{3} & \dot{q}_{0} & \dot{q}_{1} & \dot{q}_{2} & \dot{q}_{4}
\end{array}\right]^{\mathrm{T}} .
$$

The generated torque by the spring-damperelement is

$$
T(t)=-c y^{\prime}(0, t)-d \dot{y}^{\prime}(0, t)
$$

which is froportional to the joint angle $y^{\prime}(0, t)$ and to the joint angle velocity $\dot{y}^{\prime}(0, t)$ with the factors $c$ and $d$. The spring-damper-element is acting as a state feedl ack for the linear system in eq. (5) with the static feedback vector $r$ resulting in the new system

$$
\dot{x}^{\prime}(t)=\left(A-b r^{\mathrm{T}}\right) x(t) \text { and } y(t)=c^{\mathrm{T}} x(t)
$$

with the $s !$ stem matrix

$$
A_{r}(c, d)=A-b r^{\top}
$$

depending on the spring stiffness $c$ and the damping constant $d$

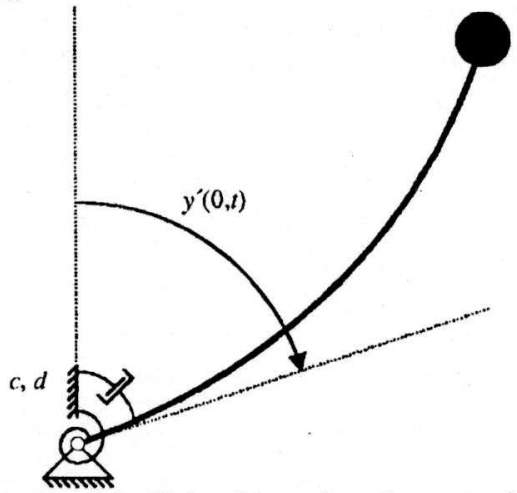

Figure 2: Flexible link with spring-damper-element

The influence of the spring-damper-element on the properties of the new system (eq. (8)) can be investigated by determing the variation of the eigenvalues of the new system (eq. (9)). For details see [2]. Fi zure 3 shows the influence of the springdamper-elt:ment for the endeffector position, when the endeff ctor ist stimulated with an impulse force. For the fired joint, a lightly damped vibration is visible wit 1 the influence of the passive mechanical element th ? vibration is atenuated. 


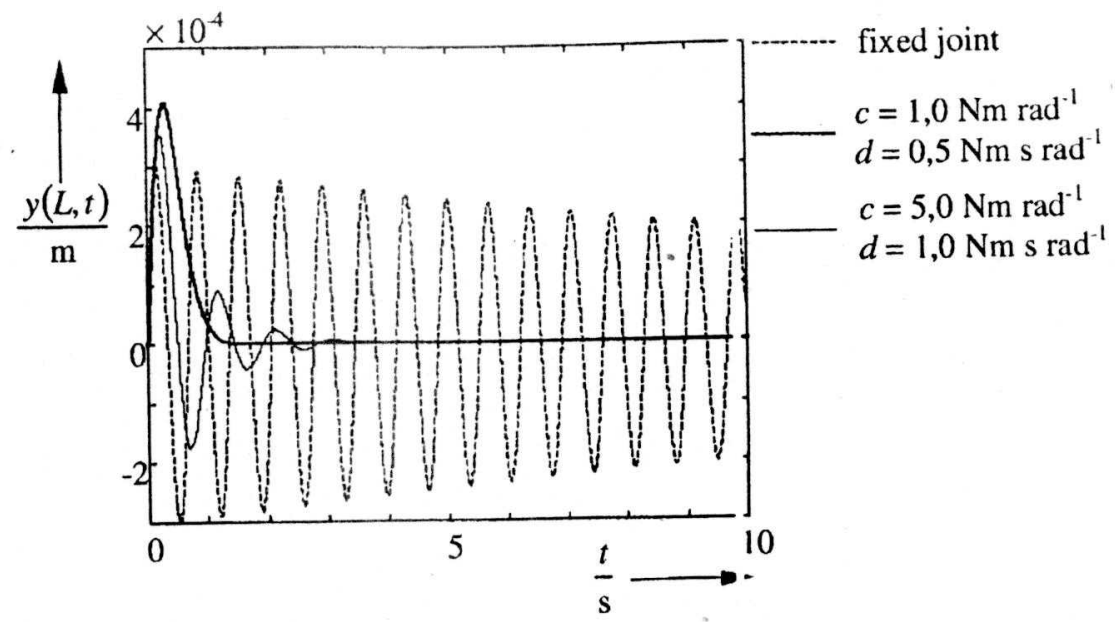

Figure 3: Position of tip mass for impulse fo:ce at the endeffector

In the case of the concrete pump the actuator is treated as a virtual passive spring-damper-element. The result is a new system with new properties depending on the spring stiffness $c$ and the damping constant $d$. The position $y_{\mathrm{Cyl}}$ of the cylinder and the force $F_{\text {Cyl }}$ acting on the piston rod are measured. The robot arm can be driven by adjusting the spring base $y_{0}$ as illustrated in figure 4. To realize the virtual spring-damper, a piston velocity setvalue for the cylinder

$$
\dot{y}_{\mathrm{Cy} 1 ., \mathrm{set}}(t)=-\frac{F_{\mathrm{Cyl}}(t)-c\left(y_{0}(t)-y_{\mathrm{C} y 1}(t)\right)}{d}
$$

has to be controlled.

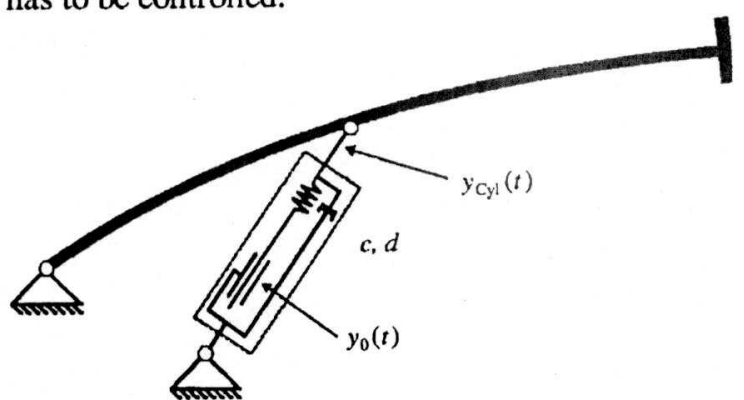

Figure 4: Flexible robot with a spring-damperelement and variation of the spring base

\section{CONTROL CONCEPT OF THE DIFFERENTIAL CYLINDER}

Hydrostatic differential cylinders have strong nonlinear static and dynamic effects. One basic approach to controlling nonlinear systems is the input/output linearization [6], [8], but for differential cylinders it is difficult to apply for online control. For practical implementation a simplified cascaded nonlinear control scheme is given in [1]. With some simplifications, two simple models for positive and negative valve inputs $u$ can be developed. The resulting nodel for positive input $u>0$

$\left[\begin{array}{c}\dot{p}_{\mathrm{A}} \\ \dot{p}_{\mathrm{B}}\end{array}\right]=\left[\begin{array}{l}\frac{E_{\mathrm{O} \text { il }}}{V} B_{\mathrm{V}} \sqrt{p_{0}-p_{\mathrm{A}}} \\ -\frac{E_{\mathrm{Oil}}}{V} B_{\mathrm{V}} \sqrt{p_{\mathrm{B}}-p_{\mathrm{T}}}\end{array}\right] u+\left[\begin{array}{c}-\frac{E_{\mathrm{Oil}}}{V} A_{\mathrm{P}} \\ \frac{E_{\mathrm{Oil}}}{V} \frac{A_{\mathrm{P}}}{\varphi}\end{array}\right] \dot{y}_{\mathrm{Cyl}}$

and the $\mathrm{m}$ odel for negative inputs $\mathrm{u}<0$

$$
\left[\begin{array}{c}
\dot{p}_{\mathrm{A}} \\
\dot{p}_{\mathrm{B}}
\end{array}\right]=\left[\begin{array}{l}
\frac{E_{\mathrm{Oil}}}{V} B_{\mathrm{V}} \sqrt{p_{\mathrm{A}}-p_{\mathrm{T}}} \\
-\frac{E_{\mathrm{Oil}}}{V} B_{\mathrm{V}} \sqrt{p_{0}-p_{\mathrm{B}}}
\end{array}\right] u+\left[\begin{array}{l}
-\frac{E_{\text {Oil }}}{V} A_{\mathrm{p}} \\
\frac{E_{\mathrm{O} i l}}{V} \frac{A_{\mathrm{P}}}{\varphi}
\end{array}\right] \dot{y}_{\mathrm{Cyl}} .
$$

For constant cylinder velocity the pressure dynamics can be neglected

$$
\dot{p}_{\mathrm{A}}=\dot{p}_{\mathrm{B}}=0
$$

and in $n$ ost cases the tank pressure can also be neglected A static input $u$ for constant velocities can be calculit ted which depends on the pump pressure $p_{0}$ and the oil pressure in chamber $\mathrm{A}$

$$
u=\frac{A_{\mathrm{P}}}{B_{\mathrm{V}} \sqrt{p_{0}-p_{\mathrm{A}}}} \dot{y}_{\mathrm{Cyl}, \mathrm{set}}
$$

for a positive input $u>0$ and

$$
u=\frac{A_{\mathrm{P}}}{B_{\mathrm{V}} \sqrt{p_{\mathrm{A}}}} \dot{y}_{\mathrm{Cyl}, \text { set }}
$$

for negat ve inputs $u<0$, or $u$ can be calculated using the pump pressure $p_{0}$ and the oil pressure in chamber Is 


$$
u=\frac{A_{\mathrm{P}}}{\varphi B_{\mathrm{V}} \sqrt{p_{\mathrm{B}}}} \dot{y}_{\mathrm{Cyl}, \text { set }}
$$

for positive inputs $u>0$ and

$$
u=\frac{A_{\mathrm{P}}}{\varphi B_{\mathrm{V}} \sqrt{p_{0}-p_{\mathrm{B}}}} \dot{y}_{\text {Cylset }}
$$

for negative inputs $u<0$ to be switched depending on the sign of $\dot{y}_{\text {Cylset }}$.

The operating curve of the valve has also been measured. Most valves in use have a measured curve differing to the theoretical. This nonlinear offset is also implemented into the velocity controller. An additional output error integration is added as depicted in figure 5. Figure 6 shows the block diagram of the entire vibration damping controller.

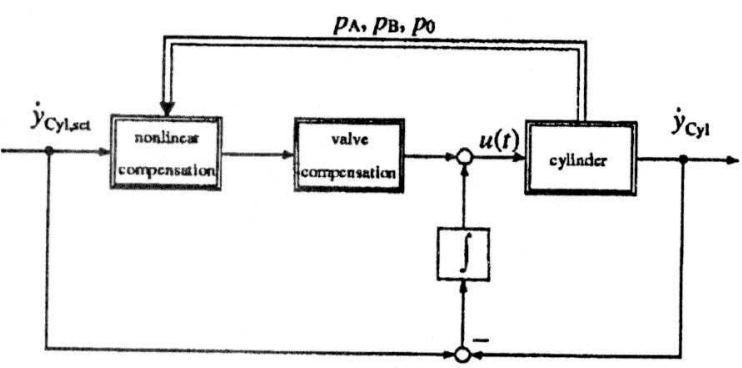

Figure 5: Velocity controller

\section{STRUCTURE OF A CONCRETE PUMP AND MEASUREMENT DEVICES}

The concrete pump consists of four arm segments and each segment is separately actuated by a differential cylinder. A redirection mechanism transforms the transverse movement into a revolving movement in the joint. The total Arm is based on the truck with rotatable joint, so that almost evry arm position is reached.

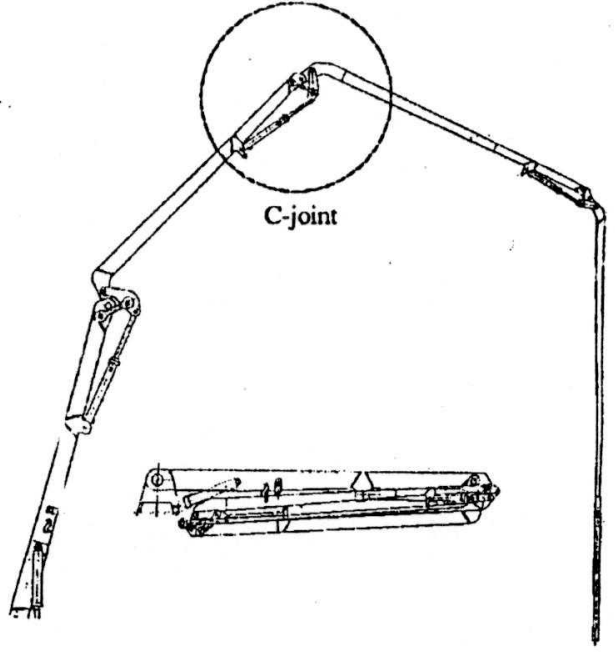

Figurs 7: Concrete pump arm in two positions

With he background of cost minimization only one actuator should be controlled to save sensors, measuren ent equipment and to make the control easy. The supposed optimum energy dissipating joint is the $\mathrm{C}$-joint (figure 7).

To retlize the control concept the conditions of the cyliniler must be known. The position of the piston rol is measured by an incremental position sensor. "hrough numerical differentiation it is possible to specify the piston velocity. Pressure sensors nieasure the pump and chamber pressures. The force $F_{\mathrm{Cyl}}$ is measured by four strain gauges, which are located at the end of the piston rod.

\section{FIRS' $[$ EXPERIMENTS WITH A REAL CONCRETE PUMP}

Figure 8 shows the measured actuator force of the cylirder in joint C. Here all links were horizontaly aligned and the endeffector was manually exited. One can see clearly the elastic link vibration of $\pm 60 \mathrm{kN}$ around a static load of about $170 \mathrm{kN}$ fading away within about $60 \mathrm{~s}$.

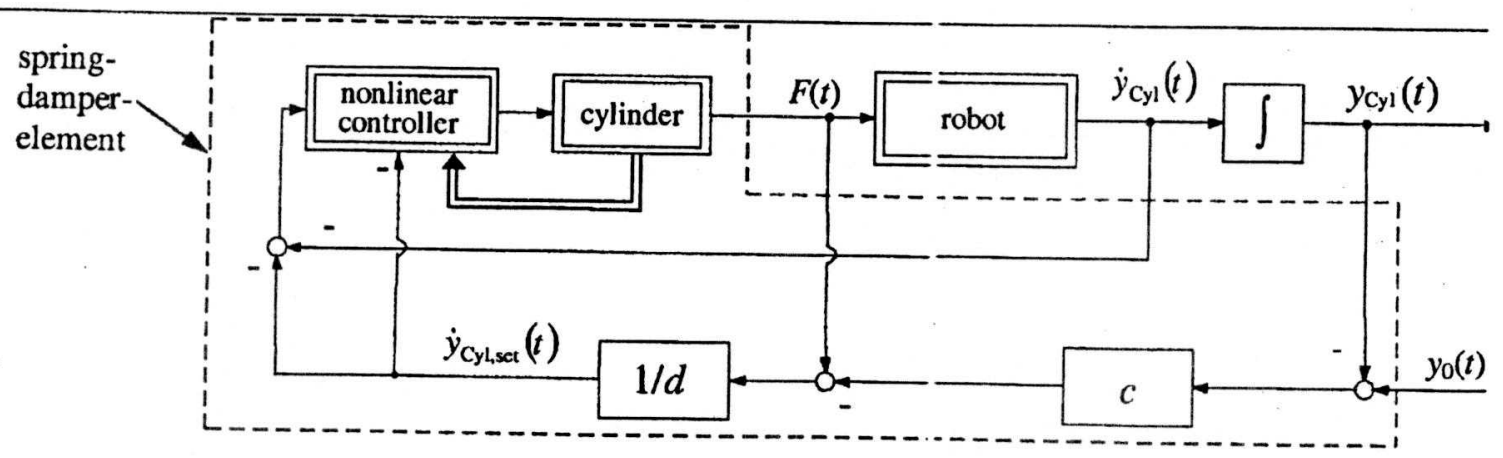

Figure 6: Block diagram of the vibration dampin y controller structure 


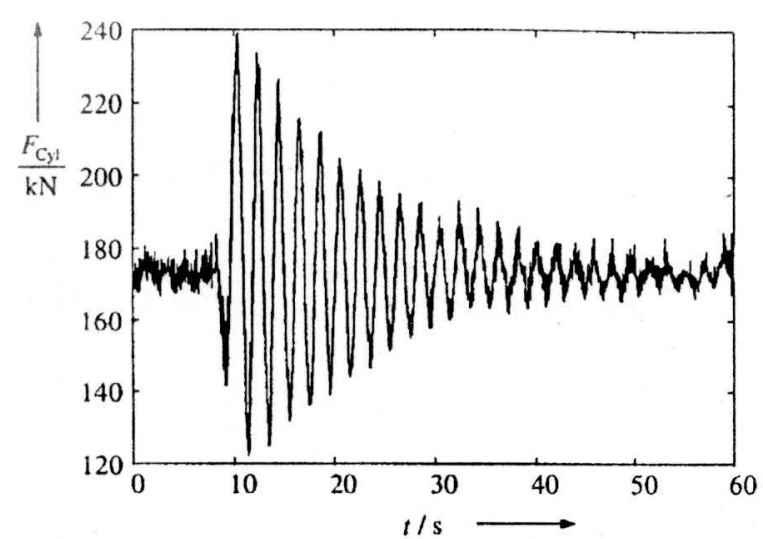

Figure 8: Measured actuator force of cylinder in joint $\mathrm{C}$

The first step before implementing the control concept into a real concrete pump robot is to verify the velocity controller. Therefore the hydrostatic differential cylinder of the concrete pump was dismounted and set up in the laboratory (see figure 9).

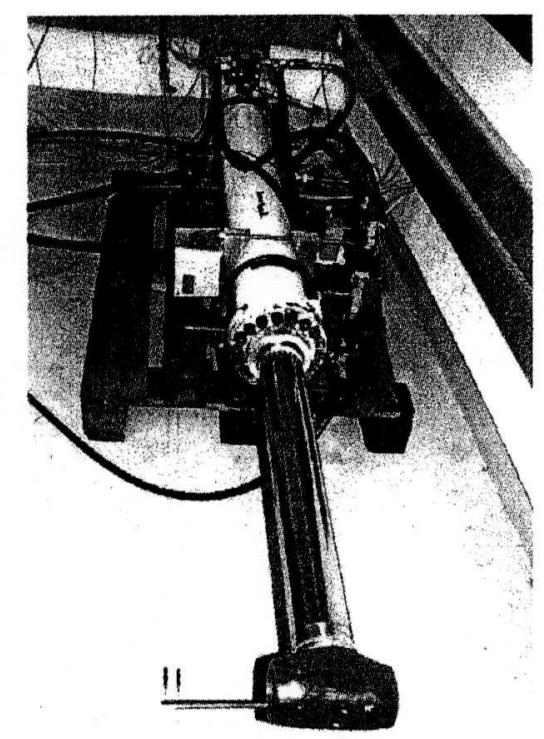

Figure 9: Dismounted hydrostatic differential cylinder of the concrete pump

A velocity controller was designed and implemented according to sections 2 and 3 . In the first experiment a sinusodial disturbance force of $\pm 60 \mathrm{kN}$ is assumed (figure 10) by a voltage source (the voltage represents a measured force). By the use of the spring-damper-element equation (10) a piston velocity set-value is calculated. Figure 11 shows the satisfactory quality of the velocity controller.

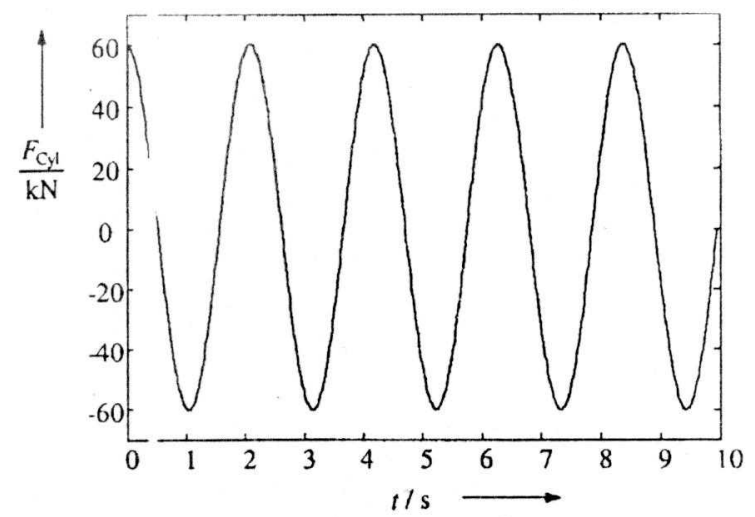

Figure 10: Actuator force

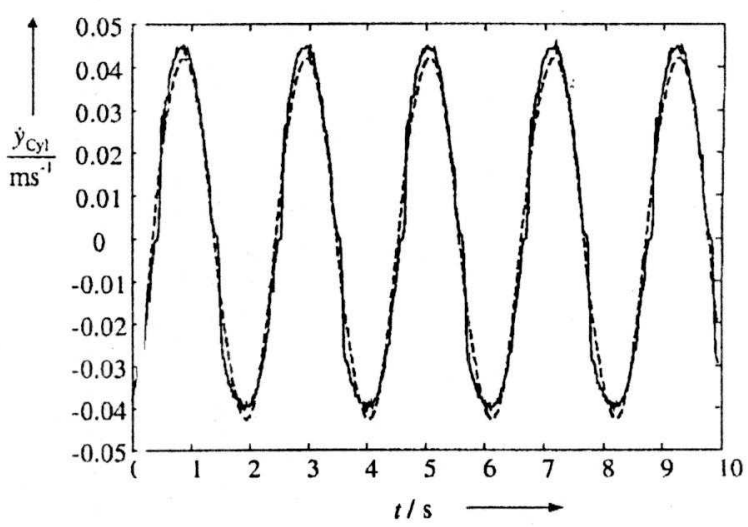

$\mathrm{Fi}_{i}$;ure 11: Piston velocity and setvalue

For thie second experiment an arbitrary force curve is generated to simulate a real input of a force sensor ( $\mathrm{f}_{i}$; rure 12$)$. Again the piston velocity setvalue is calculited by the use of the spring-damperelement ind figure 13 shows the corresponding piston vel xity including the setvalue.

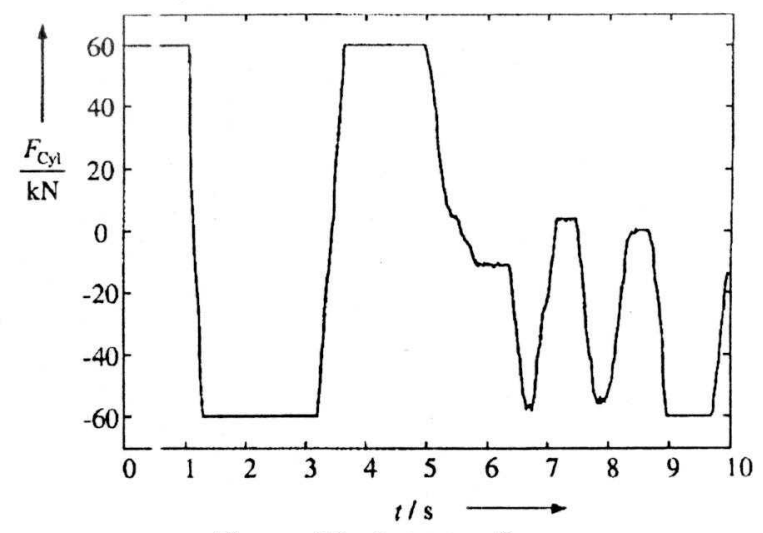

Figure 12: Actuator force

For tie final stage of the experiment this hydrostatic differential cylinder was reinstalled in the concrete pump and the control concept was proved or a real system. The optimum parameters spring sti fness $c$ and damping constant $d$ must be detected experimentally. 


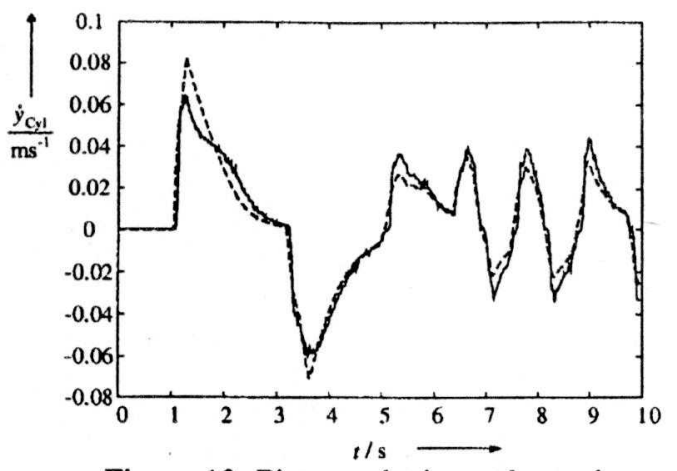

Figure 13: Piston velocity and setvalue

Figure 14 depicts the standardized actuator force of the cylinder in joint $\mathrm{C}$ (which is a measure for vibration of the endeffector) when all links were horizontally aligned and the endeffector was manually exited. In comparison to this, figure 15 shows the force curve without the controller concept for vibration damping. One can see the good vibration damping with the control concept. The vibration fades away in $5 \mathrm{~s}$. Without vibration control the vibration of the actuator force and therewith the endeffector position is fade away slowly and needs more than $20 \mathrm{~s}$. In addition the force without control is more then three times larger than the piston force with the use of the control concept.

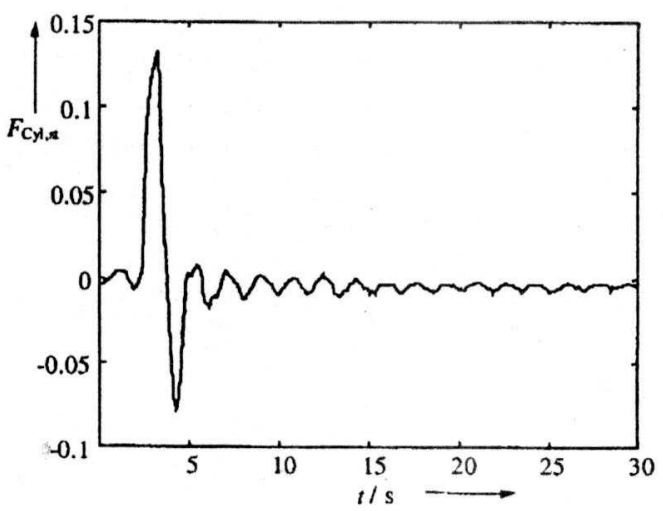

Figure 14: Actuator force with control

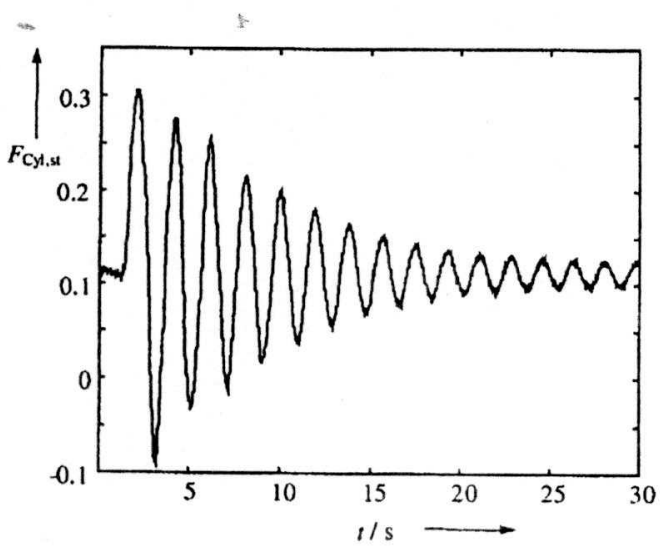

Figure 15: Actuator force without control

\section{CONCLUSION}

This paper presents the design and implementation of a recent approach to vibration damping of hydraulically driven flexible concrete pumps using virtual passive elements. The actuator is given the properties of a spring-damper element. A velocity controller realizes the passive element which always removes energy from a system, so it never can destabilize it. Hence, the controller is basically model independent; an advantage since it is not possible to establish a model for multilink flexible robots.

First the approach is implemented into an industrial concrete pump cylinder to verify the velocity controller. In the second step the actuator is installed back into the concrete pump and the control device shows excellent vibration damping of the endeffector when the arm is manually exited or moved by the joint cylinders.

In the near future the vibration control concept will be tested during a concrete pumping process. It is intended to include these results in the final version of this paper.

\section{REFERENCES}

[1] Bernzen, W., T. Wey and B. Riege. 1997. Nonlinear control of hydraulic differential cylinders actuating a flexible robot. Proc. of 36th IEEE Conference on Decision and Control (CDC'97). San Diego, CA, USA. 1333-1334.

[2] Bernzen, W. 1999. Active vibration control of flexible robots using virtual spring-damper systems. Journal of Intelligent and Robotic Systems 24(1). 69-88.

[3] Canudas de Wit, C., B. Siciliano and G. Bastin (Eds.). 1996. Theory of Robot Control. Berlin, Heidelberg: Springer.

[4] Fraser, A. R. and R. W. Daniel. 1991. Perturbation techniques for flexible manipulators. Boston: Kluwer.

[5] Heintze, J. and A. J. J. van der Weiden. 1995. Innerloop design and analysis for hydraulic actuators, with an application to impedance control. Control Engineering Practice 3. 1323-1330.

[6] Isidori, A. 1995. Nonlinear Control Systems. Berlin, Heidelberg: Springer.

[7] Meirovitch, L. 1967. Analytical Methods in Vibration. Macmillan, New-York.

[8] Schwarz, H. 1991. Nonlinear Control Systems (in German). München, Wien: Oldenbourg. 